\title{
ADAPTIVE CONTROL AND SYNCHRONIZATION OF LiU's FouR-WING CHAOTIC SYSTEM WITH CUBIC NONLINEARITY
}

\author{
Sundarapandian Vaidyanathan ${ }^{1}$ \\ ${ }^{1}$ Research and Development Centre, Vel Tech Dr. RR \& Dr. SR Technical University \\ Avadi, Chennai-600 062, Tamil Nadu, INDIA \\ sundarvtuegmail.com
}

\begin{abstract}
This paper investigates the adaptive chaos control and synchronization of Liu's four-wing chaotic system with cubic nonlinearity (Liu, 2009) and unknown parameters. First, we design adaptive control laws to stabilize the Liu's four-wing chaotic system with cubic nonlinearity to its unstable equilibrium at the origin based on the adaptive control theory and Lyapunov stability theory. Next, we derive adaptive control laws to achieve global chaos synchronization of identical Liu's four-wing chaotic systems with cubic nonlinearity and unknown parameters. Numerical simulations are shown to demonstrate the effectiveness of the proposed adaptive chaos control and synchronization schemes.
\end{abstract}

\section{KEYWORDS}

Adaptive Control, Chaos Synchronization, Chaos, Four-Wing System.

\section{INTRODUCTION}

Chaotic systems are dynamical systems that are highly sensitive to initial conditions. The sensitive nature of chaotic systems is commonly called as the butterfly effect [1]. Since chaos phenomenon in weather models was first observed by Lorenz in 1961, a large number of chaos phenomena and chaos behaviour have been discovered in physical, social, economical, biological and electrical systems.

The control of chaotic systems is to design state feedback control laws that stabilizes the chaotic systems around the unstable equilibrium points. Active control technique is used when the system parameters are known and adaptive control technique is used when the system parameters are unknown [2-4].

Synchronization of chaotic systems is a phenomenon that may occur when two or more chaotic oscillators are coupled or when a chaotic oscillator drives another chaotic oscillator. Because of the butterfly effect, which causes the exponential divergence of the trajectories of two identical chaotic systems started with nearly the same initial conditions, synchronizing two chaotic systems is seemingly a very challenging problem in the chaos literature [5-16].

In 1990, Pecora and Carroll [5] introduced a method to synchronize two identical chaotic systems and showed that it was possible for some chaotic systems to be completely synchronized. From then on, chaos synchronization has been widely explored in a variety of fields including physical systems [6], chemical systems [7], ecological systems [8], secure communications [9-10], etc. 
In most of the chaos synchronization approaches, the master-slave or drive-response formalism has been used. If a particular chaotic system is called the master or drive system and another chaotic system is called the slave or response system, then the idea of synchronization is to use the output of the master system to control the slave system so that the output of the slave system tracks the output of the master system asymptotically.

Since the seminal work by Pecora and Carroll [5], a variety of impressive approaches have been proposed for the synchronization of chaotic systems such as the sampled-data feedback synchronization method [11], OGY method [12], time-delay feedback method [13], backstepping method [14], adaptive design method [15], sliding mode control method [16], etc.

This paper is organized as follows. In Section 2, we derive results for the adaptive chaos control of the Liu's four-wing chaotic system with cubic nonlinearity (Liu, [17], 2009) and unknown parameters. In Section 3, we derive results for the adaptive synchronization of identical Liu's four-wing chaotic systems with cubic nonlinearity and unknown parameters. In Section 4, we summarize the main results obtained in this paper.

\section{Adaptive Chaos Control of LiU's Four-Wing Chaotic System WITH CUBIC NONLINEARITY}

\subsection{Theoretical Results}

The four-wing chaotic system with cubic nonlinearity (Liu, [17], 2009) is one of the important models of 3D four-wing chaotic systems. Using spectral analysis, Liu showed that this chaotic system has extremely wide frequency bandwidth compared with that of the Lorenz system and other four-wing chaotic systems.

The dynamics of Liu's four-wing system is described by

$$
\begin{aligned}
& \dot{x}_{1}=a\left(x_{2}-x_{1}\right)+x_{2} x_{3}^{2} \\
& \dot{x}_{2}=b\left(x_{1}+x_{2}\right)-x_{1} x_{3}^{2} \\
& \dot{x}_{3}=-c x_{3}+d x_{2}+x_{1} x_{2} x_{3}
\end{aligned}
$$

where $x_{1}, x_{2}, x_{3}$ are the state variables and $a, b, c, d$ are positive constants.

The system (1) is chaotic when the parameter values are taken as

$$
a=50, b=13, c=13 \text { and } d=6 .
$$

When the parameter values are taken as in (2), the system (1) is chaotic and the system linearization matrix at the equilibrium point $E_{0}=(0,0,0)$ is given by

$$
A=\left[\begin{array}{ccc}
-a & a & 0 \\
b & b & 0 \\
0 & d & -c
\end{array}\right]
$$

which has the eigenvalues 
International Journal of Computer Science, Engineering and Applications (IJCSEA) Vol.1, No.4, August 2011

$$
\lambda_{1}=-13, \lambda_{2}=-59.0247 \text { and } \lambda_{3}=22
$$

Since $\lambda_{3}>0$, it is immediate from Lyapunov stability theory [18] that the system (1) is unstable at the equilibrium point $E_{0}=(0,0,0)$. The state portrait of the chaotic system (1) is described in Figure 1.

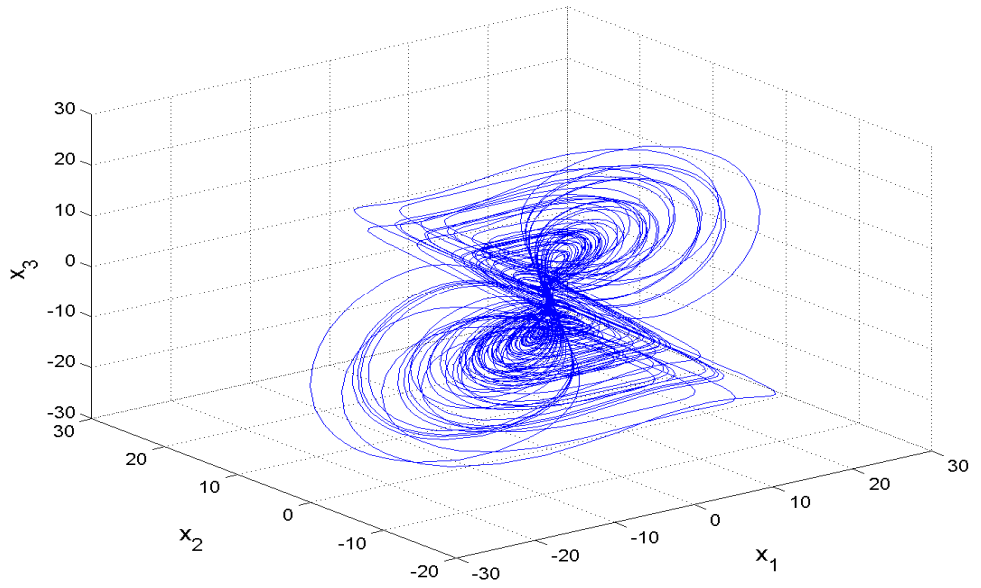

Figure 1. State Orbits of Liu's Four-Wing Chaotic System

In this section, we design adaptive control law for globally stabilizing the chaotic system (1) when the parameter values are unknown.

Thus, we consider the controlled chaotic Liu system as follows.

$$
\begin{aligned}
& \dot{x}_{1}=a\left(x_{2}-x_{1}\right)+x_{2} x_{3}^{2}+u_{1} \\
& \dot{x}_{2}=b\left(x_{1}+x_{2}\right)-x_{1} x_{3}^{2}+u_{2} \\
& \dot{x}_{3}=-c x_{3}+d x_{2}+x_{1} x_{2} x_{3}+u_{3}
\end{aligned}
$$

where $u_{1}, u_{2}$ and $u_{3}$ are feedback controllers to be designed using the states and estimates of the unknown parameters of the system.

In order to ensure that the controlled system (3) globally converges to the origin asymptotically, we consider the following adaptive control functions

$$
\begin{aligned}
& u_{1}=-\hat{a}\left(x_{2}-x_{1}\right)-x_{2} x_{3}^{2}-k_{1} x_{1} \\
& u_{2}=-\hat{b}\left(x_{1}+x_{2}\right)+x_{1} x_{3}^{2}-k_{2} x_{2} \\
& u_{3}=\hat{c} x_{3}-\hat{d} x_{2}-x_{1} x_{2} x_{3}-k_{3} x_{3}
\end{aligned}
$$

where $\hat{a}, \hat{b}, \hat{c}$ and $\hat{d}$ are estimates of the parameters $a, b, c$ and $d$, respectively, and $k_{1}, k_{2}, k_{3}$ are positive constants. 
International Journal of Computer Science, Engineering and Applications (IJCSEA) Vol.1, No.4, August 2011

Substituting the control law (4) into the Liu dynamics (1), we obtain

$$
\begin{aligned}
& \dot{x}_{1}=(a-\hat{a})\left(x_{2}-x_{1}\right)-k_{1} x_{1} \\
& \dot{x}_{2}=(b-\hat{b})\left(x_{1}+x_{2}\right)-k_{2} x_{2} \\
& \dot{x}_{3}=-(c-\hat{c}) x_{3}+(d-\hat{d}) x_{2}-k_{3} x_{3}
\end{aligned}
$$

Let us now define the parameter errors as

$$
\begin{aligned}
& e_{a}=a-\hat{a} \\
& e_{b}=b-\hat{b} \\
& e_{c}=c-\hat{c} \\
& e_{d}=d-\hat{d}
\end{aligned}
$$

Using (6), the closed-loop dynamics (5) can be written compactly as

$$
\begin{aligned}
& \dot{x}_{1}=e_{a}\left(x_{2}-x_{1}\right)-k_{1} x_{1} \\
& \dot{x}_{2}=e_{b}\left(x_{1}+x_{2}\right)-k_{2} x_{2} \\
& \dot{x}_{3}=-e_{c} x_{3}+e_{d} x_{2}-k_{3} x_{3}
\end{aligned}
$$

For the derivation of the update law for adjusting the parameter estimates $\hat{a}, \hat{b}, \hat{c}, \hat{d}$, the Lyapunov approach is used.

Consider the quadratic Lyapunov function

$$
V=\frac{1}{2}\left(x_{1}^{2}+x_{2}^{2}+x_{3}^{2}+e_{a}^{2}+e_{b}^{2}+e_{c}^{2}+e_{d}^{2}\right)
$$

which is a positive definite function on $R^{7}$.

Note also that

$$
\begin{aligned}
& \dot{e}_{a}=-\dot{\hat{a}} \\
& \dot{e}_{b}=-\dot{\hat{b}} \\
& \dot{e}_{c}=-\dot{\hat{c}} \\
& \dot{e}_{d}=-\dot{\hat{d}}
\end{aligned}
$$

Differentiating $V$ along the trajectories of (7) and using (9), we obtain

$$
\begin{aligned}
\dot{V}= & -k_{1} x_{1}^{2}-k_{2} x_{2}^{2}-k_{3} x_{3}^{2}+e_{a}\left[x_{1}\left(x_{2}-x_{1}\right)-\dot{\hat{a}}\right]+e_{b}\left[x_{2}\left(x_{1}+x_{2}\right)-\dot{\hat{b}}\right] \\
& +e_{c}\left[-x_{3}^{2}-\dot{\hat{c}}\right]+e_{d}\left[x_{2} x_{3}-\dot{\hat{d}}\right]
\end{aligned}
$$


In view of Eq. (10), the estimated parameters are updated by the following law:

$$
\begin{aligned}
& \dot{\hat{a}}=x_{1}\left(x_{2}-x_{1}\right)+k_{4} e_{a} \\
& \dot{\hat{b}}=x_{2}\left(x_{1}+x_{2}\right)+k_{5} e_{b} \\
& \dot{\hat{c}}=-x_{3}^{2}+k_{6} e_{c} \\
& \dot{\hat{d}}=x_{2} x_{3}+k_{7} e_{d}
\end{aligned}
$$

where $k_{4}, k_{5}, k_{6}, k_{7}$ are positive constants.

Substituting (11) into (10), we get

$$
\dot{V}=-k_{1} x_{1}^{2}-k_{2} x_{2}^{2}-k_{3} x_{3}^{2}-k_{4} e_{a}^{2}-k_{5} e_{b}^{2}-k_{6} e_{c}^{2}-k_{7} e_{d}^{2}
$$

which is a negative definite function on $R^{7}$.

Thus, by Lyapunov stability theory [18], we obtain the following result.

Theorem 1. The Liu's four-wing chaotic system (1) with cubic nonlinearity and unknown parameters is globally and exponentially stabilized for all initial conditions $x(0) \in R^{3}$ by the adaptive control law (4), where the update law for the parameters is given by (11) and $k_{i},(i=1, \ldots, 7)$ are positive constants.

\subsection{Numerical Results}

For the numerical simulations, the fourth order Runge-Kutta method is used to solve the chaotic system (1) with the adaptive control law (4) and the parameter update law (11).

The parameters of the Liu's four-wing system (1) are selected as

$$
a=50, \quad b=13, c=13 \text { and } d=6 .
$$

For the adaptive and update laws, we take $k_{i}=2, \quad(i=1,2, \ldots, 7)$.

Suppose that the initial values of the estimated parameters are

$$
\hat{a}(0)=12, \hat{b}(0)=8, \hat{c}(0)=6 \text { and } \hat{d}(0)=16
$$

The initial values of the Liu's four-wing system (1) are taken as $x(0)=(24,36,45)$.

When the adaptive control law (4) and the parameter update law (11) are used, the controlled Liu system converges to the equilibrium $E_{0}=(0,0,0)$ exponentially as shown in Figure 2 . The parameter estimates are shown in Figure 3. 
International Journal of Computer Science, Engineering and Applications (IJCSEA) Vol.1, No.4, August 2011

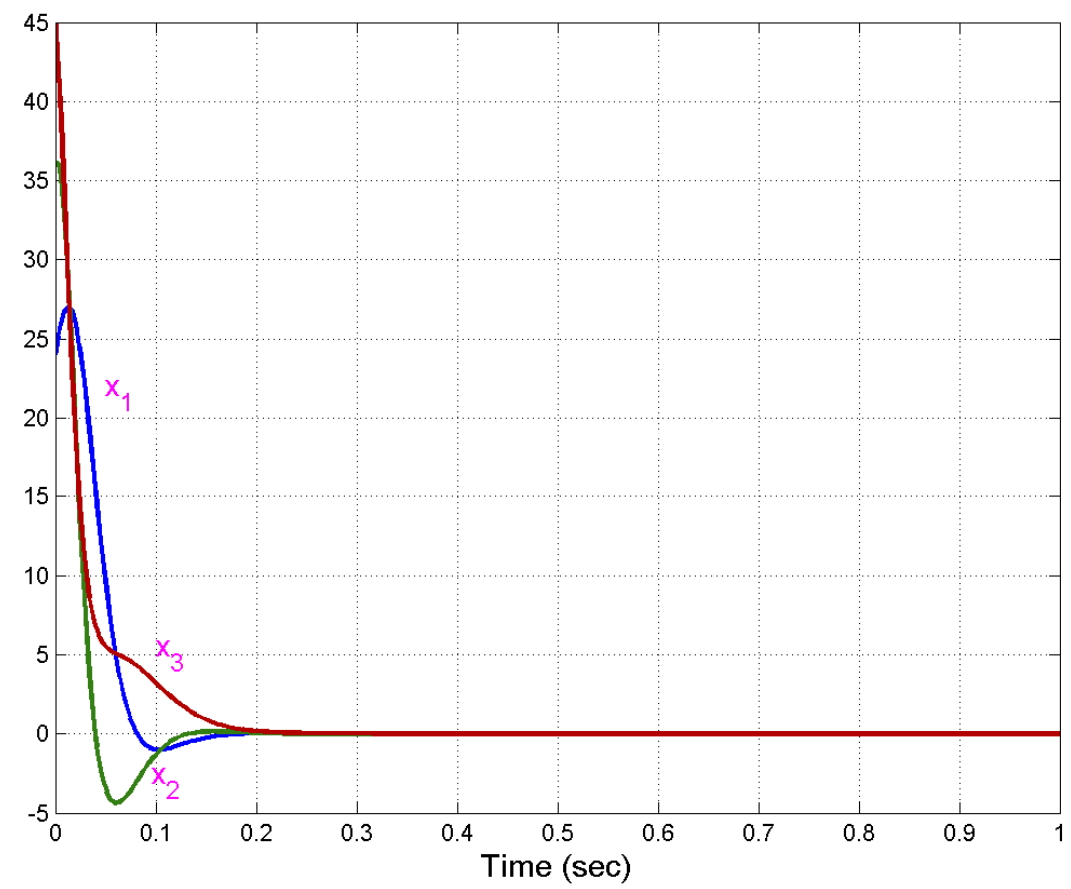

Figure 2. Time Responses of the Controlled Liu's Four-Wing System

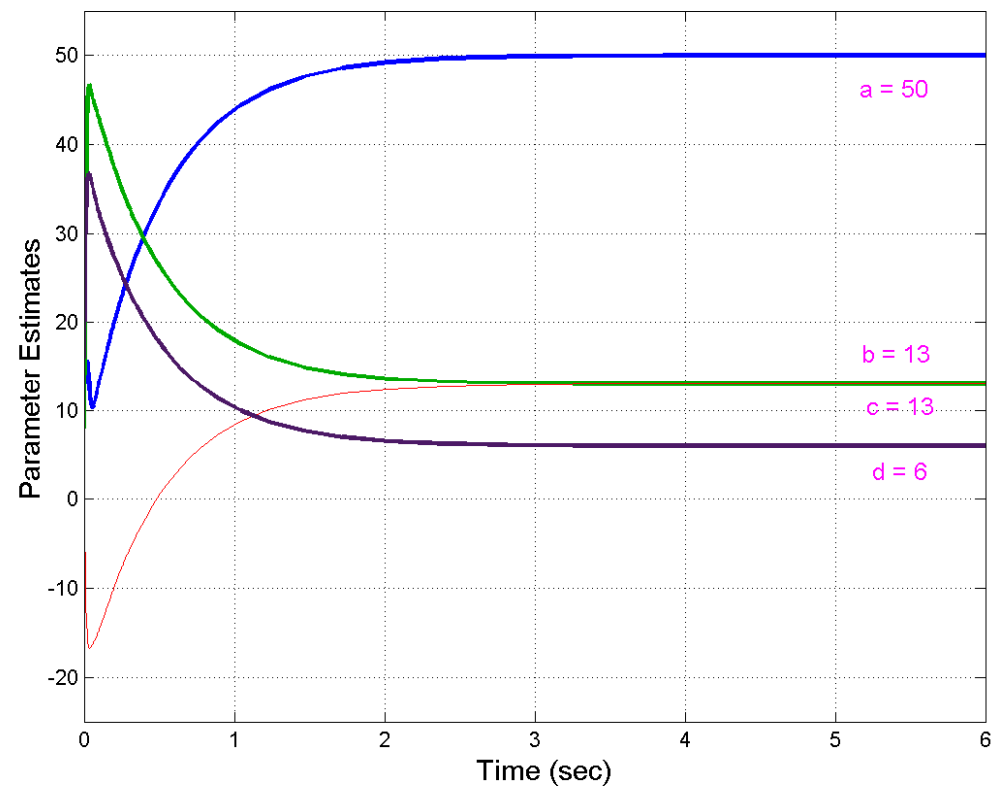

Figure 3. Parameter Estimates $\hat{a}(t), \hat{b}(t), \hat{c}(t), \hat{d}(t)$ 


\section{Adaptive Synchronization OF Identical LiU's Four-Wing CHAOTIC SYSTEMS WiTH CUBIC NONLINEARITY}

\subsection{Theoretical Results}

In this section, we discuss the adaptive synchronization of identical Liu's four-wing chaotic systems (Liu, [17], 2009) with cubic nonlinearity and unknown parameters.

As the master system, we consider the Liu's four-wing chaotic system described by

$$
\begin{aligned}
& \dot{x}_{1}=a\left(x_{2}-x_{1}\right)+x_{2} x_{3}^{2} \\
& \dot{x}_{2}=b\left(x_{1}+x_{2}\right)-x_{1} x_{3}^{2} \\
& \dot{x}_{3}=-c x_{3}+d x_{2}+x_{1} x_{2} x_{3}
\end{aligned}
$$

where $x_{1}, x_{2}, x_{3}$ are the state variables and $a, b, c, d$ are unknown system parameters.

The system (13) is chaotic when the parameter values are taken as

$$
a=50, b=13, c=13 \quad \text { and } d=6 .
$$

As the slave system, we consider the controlled Liu's four-wing chaotic system described by

$$
\begin{aligned}
& \dot{y}_{1}=a\left(y_{2}-y_{1}\right)+y_{2} y_{3}^{2}+u_{1} \\
& \dot{y}_{2}=b\left(y_{1}+y_{2}\right)-y_{1} y_{3}^{2}+u_{2} \\
& \dot{y}_{3}=-c y_{3}+d y_{2}+y_{1} y_{2} y_{3}+u_{3}
\end{aligned}
$$

where $y_{1}, y_{2}, y_{3}$ are the state variables and $u_{1}, u_{2}, u_{3}$ are the nonlinear controllers to be designed.

The synchronization error is defined by

$$
e_{i}=y_{i}-x_{i}, \quad(i=1,2,3)
$$

Then the error dynamics is obtained as

$$
\begin{aligned}
& \dot{e}_{1}=a\left(e_{2}-e_{1}\right)+y_{2} y_{3}^{2}-x_{2} x_{3}^{2}+u_{1} \\
& \dot{e}_{2}=b\left(e_{1}+e_{2}\right)-y_{1} y_{3}^{2}+x_{1} x_{3}^{2}+u_{2} \\
& \dot{e}_{3}=-c e_{3}+d e_{2}+y_{1} y_{2} y_{3}-x_{1} x_{2} x_{3}+u_{3}
\end{aligned}
$$

Let us now define the adaptive control functions $u_{1}(t), u_{2}(t), u_{3}(t)$ as

$$
\begin{aligned}
& u_{1}=-\hat{a}\left(e_{2}-e_{1}\right)-y_{2} y_{3}^{2}+x_{2} x_{3}^{2}-k_{1} e_{1} \\
& u_{2}=-\hat{b}\left(e_{1}+e_{2}\right)+y_{1} y_{3}^{2}-x_{1} x_{3}^{2}-k_{2} e_{2} \\
& u_{3}=\hat{c} e_{3}-\hat{d} e_{2}-y_{1} y_{2} y_{3}+x_{1} x_{2} x_{3}-k_{3} e_{3}
\end{aligned}
$$


International Journal of Computer Science, Engineering and Applications (IJCSEA) Vol.1, No.4, August 2011

where $\hat{a}, \hat{b}, \hat{c}$ and $\hat{d}$ are estimates of the parameters $a, b, c$ and $d$ respectively, and $k_{1}, k_{2}, k_{3}$ are positive constants.

Substituting the control law (17) into (16), we obtain the error dynamics as

$$
\begin{aligned}
& \dot{e}_{1}=(a-\hat{a})\left(e_{2}-e_{1}\right)-k_{1} e_{1} \\
& \dot{e}_{2}=(b-\hat{b})\left(e_{1}+e_{2}\right)-k_{2} e_{2} \\
& \dot{e}_{3}=-(c-\hat{c}) e_{3}+(d-\hat{d}) e_{2}-k_{3} e_{3}
\end{aligned}
$$

Let us now define the parameter errors as

$$
\begin{aligned}
e_{a} & =a-\hat{a} \\
e_{b} & =b-\hat{b} \\
e_{c} & =c-\hat{c}
\end{aligned}
$$

Substituting (19) into (18), the error dynamics simplifies to

$$
\begin{aligned}
& \dot{e}_{1}=e_{a}\left(e_{2}-e_{1}\right)-k_{1} e_{1} \\
& \dot{e}_{2}=e_{b}\left(e_{1}+e_{2}\right)-k_{2} e_{2} \\
& \dot{e}_{3}=-e_{c} e_{3}+e_{d} e_{2}-k_{3} e_{3}
\end{aligned}
$$

For the derivation of the update law for adjusting the estimates of the parameters, the Lyapunov approach is used.

Consider the quadratic Lyapunov function

$$
V=\frac{1}{2}\left(e_{1}^{2}+e_{2}^{2}+e_{3}^{2}+e_{a}^{2}+e_{b}^{2}+e_{c}^{2}+e_{d}^{2}\right)
$$

which is a positive definite function on $R^{7}$.

Note also that

$$
\begin{aligned}
& \dot{e}_{a}=-\dot{\hat{a}} \\
& \dot{e}_{b}=-\dot{\hat{b}} \\
& \dot{e}_{c}=-\dot{\hat{c}} \\
& \dot{e}_{d}=-\dot{\hat{d}}
\end{aligned}
$$

Differentiating $V$ along the trajectories of (20) and using (22), we obtain 
International Journal of Computer Science, Engineering and Applications (IJCSEA) Vol.1, No.4, August 2011

$$
\begin{aligned}
\dot{V}= & -k_{1} e_{1}^{2}-k_{2} e_{2}^{2}-k_{3} e_{3}^{2}-k_{4} e_{4}^{2}+e_{a}\left[e_{1}\left(e_{2}-e_{1}\right)-\dot{\hat{a}}\right]+e_{b}\left[e_{2}\left(e_{1}+e_{2}\right)-\dot{\hat{b}}\right] \\
& +e_{c}\left[-e_{3}^{2}-\dot{\hat{c}}\right]+e_{d}\left[e_{2} e_{3}-\dot{\hat{d}}\right]+
\end{aligned}
$$

In view of Eq. (23), the estimated parameters are updated by the following law:

$$
\begin{aligned}
& \dot{\hat{a}}=e_{1}\left(e_{2}-e_{1}\right)+k_{4} e_{a} \\
& \dot{\hat{b}}=e_{2}\left(e_{1}+e_{2}\right)+k_{5} e_{b} \\
& \dot{\hat{c}}=-e_{3}^{2}+k_{6} e_{c} \\
& \dot{\hat{d}}=e_{2} e_{3}+k_{7} e_{d}
\end{aligned}
$$

where $k_{4}, k_{5}, k_{6}, k_{7}$ are positive constants.

Substituting (24) into (23), we get

$$
\dot{V}=-k_{1} e_{1}^{2}-k_{2} e_{2}^{2}-k_{3} e_{3}^{2}-k_{4} e_{a}^{2}-k_{5} e_{b}^{2}-k_{6} e_{c}^{2}-k_{7} e_{d}^{2}
$$

which is a negative definite function on $R^{7}$.

Thus, by Lyapunov stability theory [18], it is immediate that the synchronization error and the parameter error decay to zero exponentially with time for all initial conditions.

Hence, we have proved the following result.

Theorem 2. The identical Liu's four-wing systems (13) and (14) with cubic nonlinearity and unknown parameters are globally and exponentially synchronized for all initial conditions by the adaptive control law (17), where the update law for parameters is given by (24) and $k_{i},(i=1, \ldots, 7)$ are positive constants.

\subsection{Numerical Results}

For the numerical simulations, the fourth order Runge-Kutta method is used to solve the two systems of differential equations (13) and (14) with the adaptive control law (17) and the parameter update law (24).

In the chaotic case, the parameters of the Liu's four-wing chaotic systems (13) and (14) are $a=50, b=13, c=13$ and $d=6$,

We take the positive constants $k_{i},(i=1, \ldots, 7)$ as $k_{i}=2$ for $i=1,2, \ldots, 7$.

Suppose that the initial values of the estimated parameters are $\hat{a}(0)=2, \hat{b}(0)=6, \hat{c}(0)=4$ and $\hat{d}(0)=12$.

We take the initial values of the master system $(13)$ as $x(0)=(25,15,20)$. 
International Journal of Computer Science, Engineering and Applications (IJCSEA) Vol.1, No.4, August 2011

We take the initial values of the slave system $(14)$ as $y(0)=(12,36,4)$.

Figure 4 shows the adaptive chaos synchronization of the identical Liu's four-wing chaotic systems. Figure 5 shows that the estimated values of the parameters $\hat{a}, \hat{b}, \hat{c}$ and $\hat{d}$ converge to the system parameters $a=50, b=13, c=13$ and $d=6$.
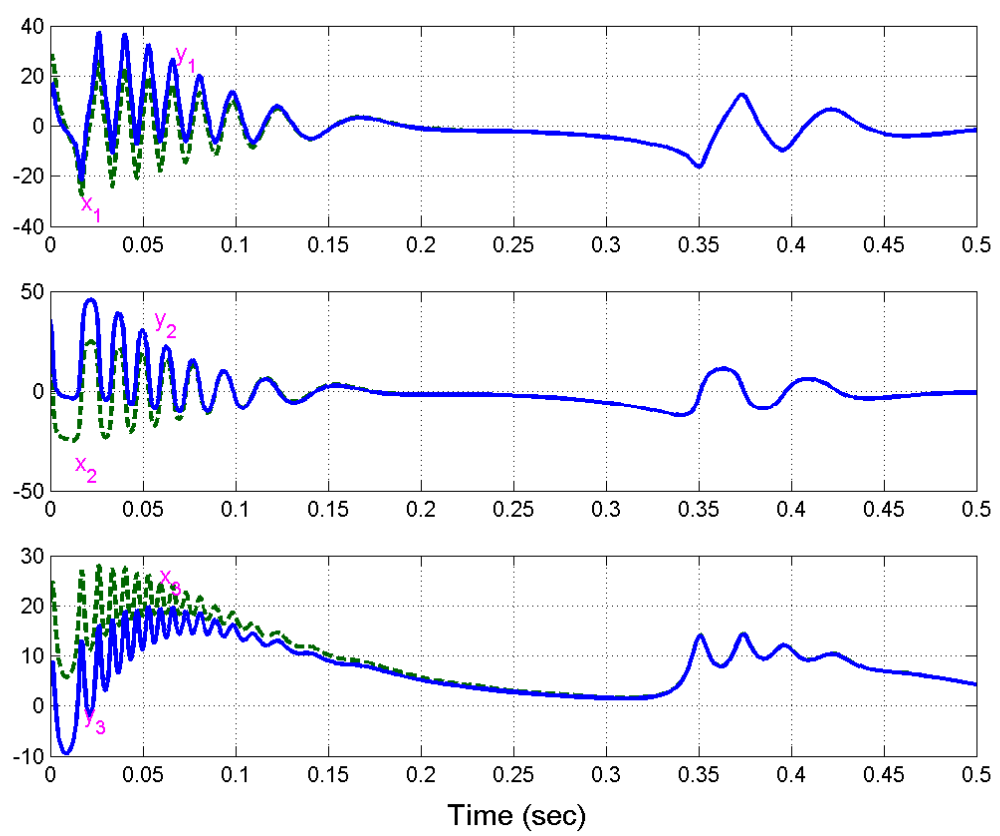

Figure 4. Adaptive Synchronization of the Identical Liu's Four-Wing Systems

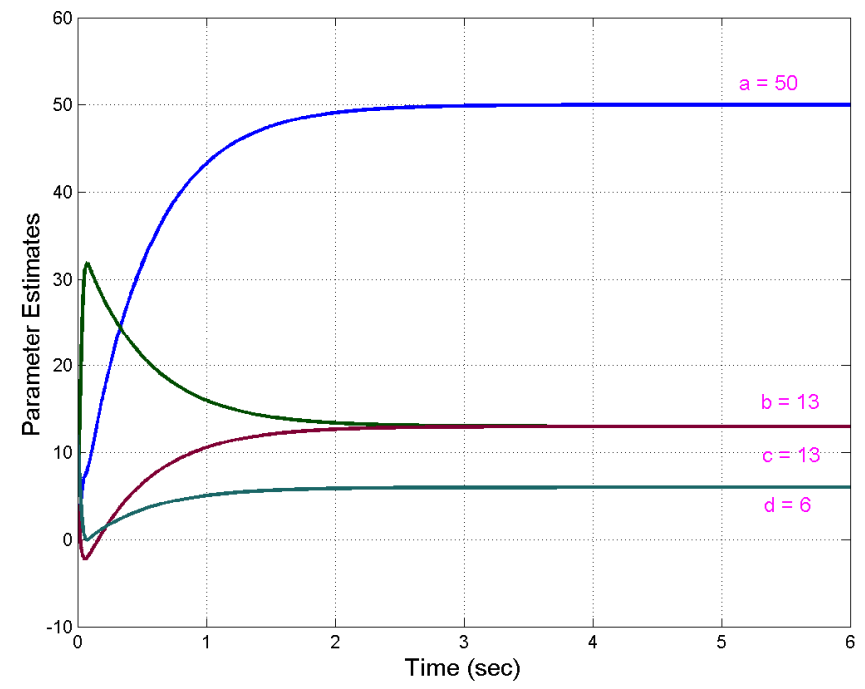

Figure 5. Parameter Estimates $\hat{a}(t), \hat{b}(t), \hat{c}(t), \hat{d}(t)$ 


\section{Conclusions}

In this paper, we deployed adaptive control theory for the chaos control and synchronization of the Liu's four-wing chaotic system (Liu, 2009) with cubic nonlinearity and unknown system parameters. First, we designed adaptive control laws to stabilize the Liu's four-wing system to its unstable equilibrium point at the origin based on the adaptive control theory and Lyapunov stability theory. Then we derived adaptive synchronization scheme and update law for the estimation of system parameters for identical Liu's four-wing chaotic systems with cubic nonlinearity and unknown parameters. Our synchronization schemes were established using Lyapunov stability theory. Since the Lyapunov exponents are not required for these calculations, the proposed adaptive control theory method is very effective and convenient to achieve chaos control and synchronization of Liu's four-wing chaotic systems. Numerical simulations are shown to demonstrate the effectiveness of the proposed adaptive chaos control and synchronization schemes.

\section{REFERENCES}

[1] Alligood, K.T., Sauer, T. \& Yorke, J.A. (1997) Chaos: An Introduction to Dynamical Systems, Springer, New York.

[2] Ge, S.S., Wang, C. \& Lee, T.H. (2000) "Adaptive backstepping control of a class of chaotic systems," Internat. J. Bifur. Chaos, Vol. 10, pp 1149-1156.

[3] Wang, X., Tian, L. \& Yu, L. (2006) "Adaptive control and slow manifold analysis of a new chaotic system,” Internat. J. Nonlinear Science, Vol. 21, pp 43-49.

[4] Sun, M., Tian, L., Jiang, S. \& Xun, J. (2007) "Feedback control and adaptive control of the energy resource chaotic system," Chaos, Solitons \& Fractals, Vol. 32, pp 168-180.

[5] Pecora, L.M. \& Carroll, T.L. (1990) "Synchronization in chaotic systems", Phys. Rev. Lett., Vol. 64, pp 821-824.

[6] Lakshmanan, M. \& Murali, K. (1996) Nonlinear Oscillators: Controlling and Synchronization, World Scientific, Singapore.

[7] Han, S.K., Kerrer, C. \& Kuramoto, Y. (1995) "Dephasing and bursting in coupled neural oscillators", Phys. Rev. Lett., Vol. 75, pp 3190-3193.

[8] Blasius, B., Huppert, A. \& Stone, L. (1999) "Complex dynamics and phase synchronization in spatially extended ecological system”, Nature, Vol. 399, pp 354-359.

[9] Feki, M. (2003) "An adaptive chaos synchronization scheme applied to secure communication", Chaos, Solitons and Fractals, Vol. 18, pp 141-148.

[10] Murali, K. \& Lakshmanan, M. (1998) "Secure communication using a compound signal from generalized synchronizable chaotic systems", Phys. Rev. Lett. A, Vol. 241, pp 303-310.

[11] Yang, T. \& Chua, L.O. (1999) "Control of chaos using sampled-data feedback control”, Internat. J. Bifurcat. Chaos, Vol. 9, pp 215-219.

[12] Ott, E., Grebogi, C. \& Yorke, J.A. (1990) “Controlling chaos”, Phys. Rev. Lett., Vol. 64, pp 11961199.

[13] Park, J.H. \& Kwon, O.M. (2003) "A novel criterion for delayed feedback control of time-delay chaotic systems”, Chaos, Solitons and Fractals, Vol. 17, pp 709-716.

[14] Yu, Y.G. \& Zhang, S.C. (2006) "Adaptive backstepping synchronization of uncertain chaotic systems”, Chaos, Solitons and Fractals, Vol. 27, pp 1369-1375.

[15] Liao, T.L. \& Tsai, S.H. (2000) "Adaptive synchronization of chaotic systems and its applications to secure communications", Chaos, Solitons and Fractals, Vol. 11, pp 1387-1396. 
International Journal of Computer Science, Engineering and Applications (IJCSEA) Vol.1, No.4, August 2011

[16] Konishi, K.., Hirai, M. \& Kokame, H. (1998) "Sliding mode control for a class of chaotic systems", Phys. Lett. A, Vol. 245, pp 511-517.

[17] Liu, X. (2009) "A new four-wing chaotic system with cubic nonlinearity and its circuit implementation,” Chin. Phys. Lett., Vol. 26, pp 504-507.

[18] Hahn, W. (1967) The Stability of Motion, Springer, New York.

\section{Author}

Dr. V. Sundarapandian is a Professor (Systems and Control Engineering), Research and Development Centre at Vel Tech Dr. RR \& Dr. SR Technical University, Chennai, India. His current research areas are: Linear and Nonlinear Control Systems, Chaos Theory, Dynamical Systems and Stability Theory, Soft Computing, Operations Research, Numerical Analysis and Scientific Computing, Population Biology, etc. He has published over 170 research articles in international journals and two text-books with Prentice-Hall of India, New Delhi, India. He has published over 50 papers in International Conferences and 90 papers in National Conferences. He is the Editor-in-Chief of the AIRCC journals - International Journal of Instrumentation and Control Systems, International Journal of Control Systems and Computer Modelling. He has delivered several Key Note Lectures on Control Systems, Chaos Theory, Scientific Computing, SCILAB, etc.

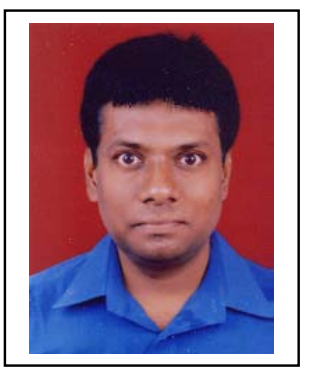

